Proceedings of the 1994 IEEE International Conference on Image Processing (ICIP-94), pp. 530-534. (Austin, Texas, 13-16 November 1994.)

\title{
A MORPHOLOGY-BASED FiLter STRUCTURE FOR EDGE-ENHANCING SMOOTHING
}

\author{
Mark A. Schulze and John A. Pearce \\ Department of Electrical Engineering and Biomedical Engineering Program \\ Engineering Science Building 610 \\ The University of Texas at Austin \\ Austin, Texas 78712
}

\begin{abstract}
We introduce the value-and-criterion filter structure, a new framework for designing filters based on mathematical morphology. The value-and-criterion filter structure is more flexible than the morphological structure, because it allows linear and nonlinear operations other than just the minimum and maximum to be performed on the data. One particular value-and-criterion filter, the Mean of Least Variance (MLV) filter, finds the mean over the "subwindow" of data with the smallest variance within an overall window. The ability of the MLV filter to smooth noise while preserving and enhancing edges and corners is demonstrated. An example application of the MLV filter in improving the contrast of magnetic resonance images is also shown.
\end{abstract}

\section{INTRODUCTION}

This paper introduces a new framework for image filtering, the value-and-criterion filter structure, which is based on the concepts of mathematical morphology. The morphological opening and closing operators exhibit a regular subwindow structure that results naturally from the sequential application of operations in a sliding filter window, or structuring element. This subwindow structure is the basis for the value-and-criterion filter structure, which allows the use of both linear and nonlinear operations with the shape-based constructs of mathematical morphology.

The morphological opening and closing operators are each made up of two sequential ordering operations. Opening is a sliding minimum operation (erosion) followed by a sliding maximum operation (dilation); closing is dilation followed by erosion. At each point in an image, the opening is the maximum of several minima, and the closing is the minimum of several maxima. A value-and-criterion filter also gets its output from an operator acting on the results of a first stage. However, instead of a single first operator such as erosion or dilation, a value-and-criterion filter has two different operations in the first stage. One of the first-stage operators (the criterion operator) provides information used to decide which of the values from the other first-stage operator (the value operator) will be the final output. This new filter structure is significant because it unifies many different types of filters with both linear and nonlinear operators into a single mathematical formalism.

The idea of choosing among several local neighborhoods based on a criterion other than the output of the filtering operation has been used many times before in schemes for edge-preserving smoothing [1-5]. Typically, these smoothing algorithms output the mean of a local neighborhood that has the smallest variance. For example, Kuwahara [1] defined a smoothing filter that finds the quadrant of a filtering window that has the smallest sample variance, and outputs the mean of that quadrant. The idea behind this smoothing technique is that regions containing edges will have a higher variance than more homogeneous regions, and so the mean is taken over smooth regions and not across edges.

In the value-and-criterion filter structure, letting the value function be the mean and the criterion function be the variance yields a filter similar to the edge-preserving filters described above. We call this filter the Mean of Least Variance (MLV) filter. The MLV filter has a more regular structure of local neighborhoods and examines more such neighborhoods than the earlier filters. The value-and-criterion filter structure also suggests an efficient way to compute the filters, since the two operations in the first stage may be performed independently.

\section{THE VALUE-AND-CRITERION FILTER STRUCTURE}

The value-and-criterion filter structure has two functions which act on the original image $f(\mathbf{x})$. These functions are the "value" function, $V$, and the "criterion" function, $C$, and are defined over a structuring element $N$. The "selection" operator, $S$, acts on the output of the criterion function and is defined over the structuring element $\tilde{N}$, a $180^{\circ}$ rotation of $N$. If $g(\mathbf{x})$ denotes the output of a valueand-criterion filter, and $v(\mathbf{x})$ and $c(\mathbf{x})$ denote the output of $V$ and $C$, respectively, then the output of the filter is defined by equations (1)-(3) below. 


$$
\begin{gathered}
v(\mathbf{x})=V\{f(\mathbf{x}) ; N\} \\
c(\mathbf{x})=C\{f(\mathbf{x}) ; N\} \\
g(\mathbf{x})=v\left(\left\{\mathbf{x}^{\prime}: \mathbf{x}^{\prime} \in \quad \quad ; \quad c\left(\mathbf{x}^{\prime}\right)=S\{c(\mathbf{x}) ; \quad\}\right\}\right)
\end{gathered}
$$

where $\tilde{N}_{\mathbf{X}}$ denotes the translation of $\tilde{N}$ such that it is centered at position $\mathbf{x}$.

Note that more than one value of $\mathbf{x}^{\prime}$ may be chosen by the selection operator if two or more values of the criterion function in the window are equal. In this case, the above definition may give more than one value of $v(\mathbf{x})$ for output. Some method of deciding among these values is required. Two potential solutions are: (1) to average all the selected values of $v\left(\mathbf{x}^{\prime}\right)$ to yield the final output, and (2) to choose the value of $v\left(\mathbf{x}^{\prime}\right)$ closest to the value of $f(\mathbf{x})$ (that is, such that $\left|f(\mathbf{x})-v\left(\mathbf{x}^{\prime}\right)\right|$ is minimum) as the final output, settling ties consistently in favor of either the higher $\left[v\left(\mathbf{x}^{\prime}\right)>f(\mathbf{x})\right]$ or lower $\left[v\left(\mathbf{x}^{\prime}\right)<f(\mathbf{x})\right]$ value. The examples in this work use the second solution, and settle ties in favor of the higher value. This solution is less resistant to noise at $f(\mathbf{x})$ than the first solution, but performs better at sharp, noiseless edges (where ties are likely). The first solution provides better noise reduction in some situations, but sometimes blurs sharp edges slightly.

Morphological opening is a value-and-criterion filter where $V$ and $C$ are both the minimum operator and $S$ is the maximum operator. Similarly, closing is a value-andcriterion filter where $V$ and $C$ are the maximum operator and $S$ is the minimum operator. New filters can be designed with the value-and-criterion structure where $V$ and $C$ are not the same. An example of one of these new filters is the Mean of Least Variance (MLV) filter discussed below.

An important difference between the subwindow structure of the value-and-criterion filters and that of the earlier edge-preserving smoothing filters is the number and regularity of the subwindows within the overall filter window. In the edge-preserving smoothing filter structures described previously, there are fewer subwindows, or the subwindows are irregularly shaped and/or differently sized. In the value-and-criterion structure with an $n \times n$ square structuring element, there are $n^{2}$ subwindows within a square overall window of size $(2 n-1) \times(2 n-1)$. Thus, the number of subwindows within the overall window grows quickly as the size of the structuring element increases. Because the previous filter structures were not as computationally efficient as the value-and-criterion structure, they only considered several (often four, but sometimes as many as seven) subwindows, and the subwindows were only defined for a particular window shape. The value-andcriterion structure, however, identifies all the subwindows of the same shape and size within an overall window. (In fact, the overall window of a value-and-criterion filter is defined by the subwindows.) Yet because of their more efficient definition, value-and-criterion filters execute considerably faster than previous implementations of related smoothing filters.

One way that is often convenient for implementing a value-and-criterion filter on a computer is to form a complex image where the real part consists of the output of the criterion stage, and the imaginary part contains the output of the value stage of the filter. The selection operator is then applied only to the real part of the complex image (while still keeping the imaginary part attached), and the filter output is the imaginary part of the result of the selection operator. This is merely a way to avoid explicitly indexing the output of the value operator separately from that of the criterion operator, but it considerably improves the speed of the algorithm in some high-level languages and image processing applications.

\section{MEAN OF LEAST VARIANCE (MLV) FILTER}

\subsection{Definition}

The Mean of Least Variance (MLV) filter is a standard value-and-criterion filter where the value function is the sample mean, the criterion function is the sample variance, and the selection operator is the minimum. Since the sample variance requires the sample mean for its computation, the $V$ and $C$ functions are not really independent in this case. The MLV filter is described by equations (4)-(6) below.

$$
\begin{gathered}
v(\mathbf{x})=\frac{1}{|N|} \sum_{\mathbf{y} \in N_{\mathbf{x}}} f(\mathbf{y}) \\
c(\mathbf{x})=\frac{1}{|N|} \sum_{\mathbf{y} \in N_{\mathbf{x}}}|f(\mathbf{y})-v(\mathbf{x})|^{2} \\
\operatorname{MLV}\{f(\mathbf{x}) ; N\}= \\
v\left(\left\{\mathbf{x}^{\prime}: \mathbf{x}^{\prime} \in \tilde{N}_{\mathbf{x}} ; c\left(\mathbf{x}^{\prime}\right)=\min \left[c(\mathbf{y}): \mathbf{y} \in \tilde{N}_{\mathbf{x}}\right]\right\}\right)
\end{gathered}
$$

Note that the $\frac{1}{|N|}$ term in the definition of $c(\mathbf{x})$ is a normalization constant and does not affect the selection operator (minimum); therefore, it may be removed from equation (5) without changing the operation of the filter. Also, recall that if several values of $\mathbf{x}^{\prime}$ satisfy the selection criterion, the value of $v\left(\mathbf{x}^{\prime}\right)$ closest to $f(\mathbf{x})$ is chosen, with ties settled in favor of higher values.

The MLV filter acts on an effective window of $W=N \oplus \tilde{N}$, where $\oplus$ denotes morphological dilation. For example, a 1-D structuring element $N$ of length 5 leads to an effective window $W$ of length 9, and a 2-D square structuring element of size $3 \times 3$ produces an overall window that is $5 \times 5$ square. The MLV filter outputs the sample mean of the subwindow $N$ with the smallest variance within the overall window $W$. This filter is similar to those described in [1-5], but its subwindow structure is more thorough than any of the previous filters. The number of subwindows $N$ in the overall window $W$ is 
equal to the number of points in $N$. The value-andcriterion structure also suggests a very efficient implementation of the MLV filter, in contrast to the previous filters, which computed each subwindow separately for every window location. Note also that the definition of the MLV filter does not specify a dimensionality, so that 1-D, 2-D, and 3-D filters can all be defined using equations (4)-(6). Earlier filters were limited to 2-D applications.

\subsection{Properties}

The MLV filter preserves sharp edges between homogeneous regions in an image, since structuring elements (subwindows) containing an edge typically have a higher variance than those that do not. Thus, the sample mean output by the MLV filter is from a subwindow that does not contain an edge. The MLV filter also sharpens blurred edges that are not perfect steps. This is illustrated in the one-dimensional example below (Fig. 1). The structuring element $(N)$ is five points long in this example.

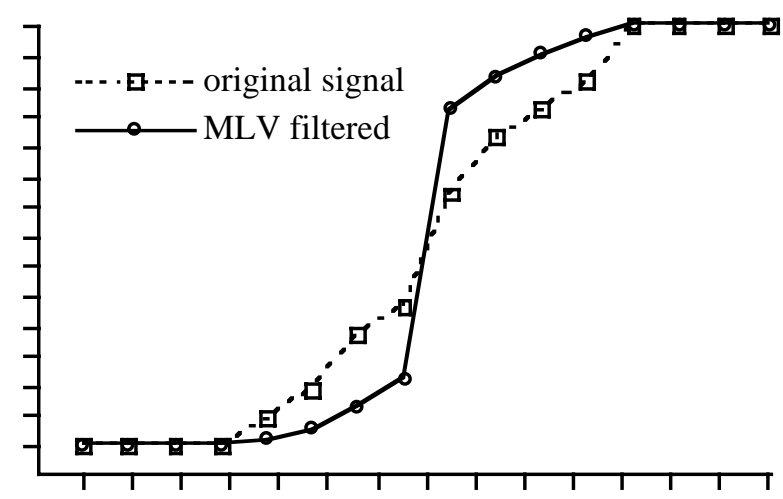

Fig. 1. Edge-enhancing effect of 1-D MLV filter $(|N|=5)$.

In addition to sharpening edges, the MLV filter with a square structuring element preserves $90^{\circ}$ corners. This is similar to the behavior of morphological filters, and is related to the shape-based aspects of the filter structure. To demonstrate the excellence of the MLV filter at preserving corners, we use the technique described in [6] to determine the corner response of the MLV filter for corners of all angles. This method computes the fraction of binary corners of various angles preserved by a filter. This fraction is found by integrating the filtered image over the area of the original binary corner within a "region of interest" the size of the overall filter window. The original corner is assumed to have its vertex at an inside corner of the region of interest, and one edge aligned with the horizontal image axis.

According to this technique, the MLV filter preserves a greater percentage of all acute binary corners than the median filter and the morphological opening. The MLV filter perfectly preserves corners of $90^{\circ}$ or more, as does the morphological opening. The corner response of the MLV filter compared to morphological opening and the median filter is shown in Fig. 2. The fractional preservation of a binary corner for each filter is plotted in polar form for angles from $-180^{\circ}$ to $+180^{\circ}$. A value of 1 indicates perfect preservation and a value of 0 indicates complete removal of the corner in the area of the filter window. The fractional preservations shown below were estimated using a $13 \times 13$ square structuring element for the MLV filter, and using a $63 \times 63$ square filter window for the median filter. The response of the morphological opening is given in a general form (for continuous space) in [6].

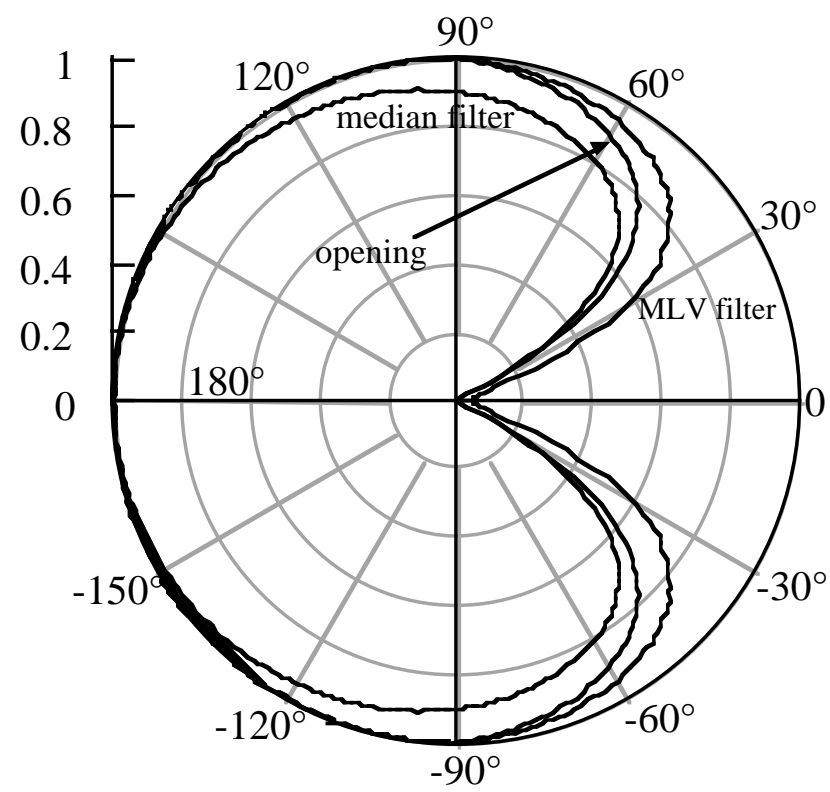

Fig. 2. Fractional corner preservation of the MLV filter, median filter, and morphological opening (square windows).

\subsection{Example}

An example of the enhancement of boundaries between homogeneous regions by the MLV filter is shown in the figures below. Fig. 3 is a magnetic resonance image (MRI) of a human head in coronal section. A common image analysis task for this type of image is segmenting the different types of tissues in the brain into different classes [7]. The MLV filter works well as a pre-filter for this problem, since it smoothes noise in homogeneous regions and sharpens the boundaries between regions.

Gerig [8] used anisotropic diffusion to reduce noise and improve the contrast of magnetic resonance images. These effects are similar to those of the MLV filter. One major difference is that anisotropic diffusion requires many iterations to yield a good result, whereas the MLV filter produces most of its improvement in the first pass. Both techniques require parameters to be chosen that affect the output. Anisotropic diffusion requires choosing the number of iterations and a parameter that affects the level of noise smoothing and the ability of the filter to preserve edges. For the MLV filter, the size and shape of the structuring element must be chosen. The size of the structuring element directly influences the degree of noise 
smoothing by the filter, and the size and shape both affect the preservation of fine details in the image.

Since a "noiseless original" image corresponding to the noisy MRI cannot be found, standard techniques for estimating image quality and the signal-to-noise ratio cannot be used. Gerig estimated the noise in MRIs by finding the $8 \times 8$ regions with the smallest standard deviation in the tissue and background areas of the image. The assumption behind this method is that an area with the smallest variation represents a homogeneous region of the tissue (or background) and therefore the standard deviation of this region represents the amount of noise in these regions. This method will automatically choose areas with the lowest noise as well, so the noise estimate is probably low. The same $8 \times 8$ regions are used to estimate the noise in the filtered image when comparing it to the unfiltered image.

Using this noise estimation technique, Gerig found for anisotropic diffusion that the ratio of the noise estimates of the filtered to the unfiltered image ranged between 0.15 to 0.20 for background and between 0.27 and 0.30 for tissue for images of a formalin fixed human brain [8]. This means that anisotropic diffusion reduced the estimated standard deviation of the noise in the tissue regions to onethird to one-fourth of the original value.

The results of one iteration of the MLV filter with a $5 \times 5$ structuring element on the original MR image of a human head are shown in Fig. 4. One iteration of the MLV filter with a $3 \times 3$ structuring element is shown in Fig. 5. The edges between different types of tissue are more distinct in the filtered images than in the original, and the noise in the image is reduced by filtering. Estimating the noise from the $8 \times 8$ regions with the smallest standard deviation in the tissue and background areas of the image, the ratio of the filtered to unfiltered noise for one iteration of the MLV filter with a $5 \times 5$ structuring element is 0.00 for background and 0.28 for tissue. For the background region, the MLV filter reduces the entire $8 \times 8$ region to a constant gray level (assuming the filtered image is rounded to integer gray level values). Therefore, a single pass of the $5 \times 5 \mathrm{MLV}$ filter provides noise reduction and edge enhancement similar to many passes of Gerig's anisotropic diffusion method. For the MLV filter with a $3 \times 3$ structuring element, the ratios are 0.33 for background and 0.66 for tissue, indicating slightly less noise reduction than for anisotropic diffusion. However, the smaller structuring element does preserve fine details in the image better than the $5 \times 5$ structuring element does.

Another nonlinear filter that is often used to suppress noise while preserving edges is the median filter. However, the median filter does not improve the contrast of images because it does not sharpen edges or preserve corners, unlike the MLV filter. The results of $5 \times 5$ square median filtering of the original image are shown in Fig. 6. The edges in the median-filtered image are not nearly as distinct as in the MLV-filtered image, although both filters remove noise quite well.

\section{CONCLUSIONS}

The value-and-criterion filter structure is useful for designing filters with various linear and nonlinear operators that also have the shape-based characteristics of morphological filters. Value-and-criterion filters extend easily to three dimensions, and the structure is computationally more efficient than similar filters defined previously. An important example of a value-and-criterion filter is the MLV filter, which uses the variance of local areas to determine which mean to use as the output of the filter. The MLV filter exhibits edge-enhancing noise smoothing behavior, and results in images that are significantly sharper than median-filtered images.

\section{ACKNOWLEDGMENTS}

The authors would like to thank Dr. Gilbert Hillman of the University of Texas Medical Branch in Galveston for providing the magnetic resonance images for this work.

This material is based upon work supported under a National Science Foundation Graduate Fellowship and an AT\&T Bell Laboratories Ph.D. Scholarship.

\section{REFERENCES}

1. M. Kuwahara, K. Hachimura, S. Eiho, and M. Kinoshita, "Processing of RI-angiocardiographic images," in Digital Processing of Biomedical Images, K. Preston Jr. and M. Onoe, Editors. New York: Plenum, 1976. pp. 187-202.

2. J. Y. Hsiao and A. A. Sawchuk, "Supervised textured image segmentation using feature smoothing and probabilistic relaxation techniques," IEEE Trans. Patt. Anal. Mach. Intell., vol. 11, no. 12, pp. 1279-1292, 1989.

3. F. Tomita and S. Tsuji, "Extraction of multiple regions by smoothing in selected neighborhoods," IEEE Trans. Syst., Man, Cyber., vol. 7, pp. 107-109, 1977.

4. M. Nagao and T. Matsuyama, "Edge preserving smoothing," Comp. Graphics Image Process., vol. 9, pp. 394-407, 1979.

5. J.-S. Lee, "Refined filtering of image noise using local statistics," Comp. Graphics Image Process., vol. 15, pp. 380-389, 1981.

6. M. A. Schulze and J. A. Pearce, "A new method for quantifying the response of filters at corners," in Nonlinear Image Processing $V$, E. R. Dougherty, J. Astola, and H. G. Longbotham, Editors, Proc. SPIE, vol. 2180, pp. 78-89, 1994

7. M. E. Brummer, R. M. Mersereau, R. L. Eisner, and R. R. J. Lewine, "Automatic detection of brain contours in MRI data sets," IEEE Trans. Med. Imag., vol. 12, no. 2, pp. 153-166, 1993.

8. G. Gerig, O. Kübler, R. Kikinis, and F. A. Jolesz, "Nonlinear anisotropic filtering of MRI data," IEEE Trans. Med. Imag., vol. 11, no. 2, pp. 221-232, 1992. 


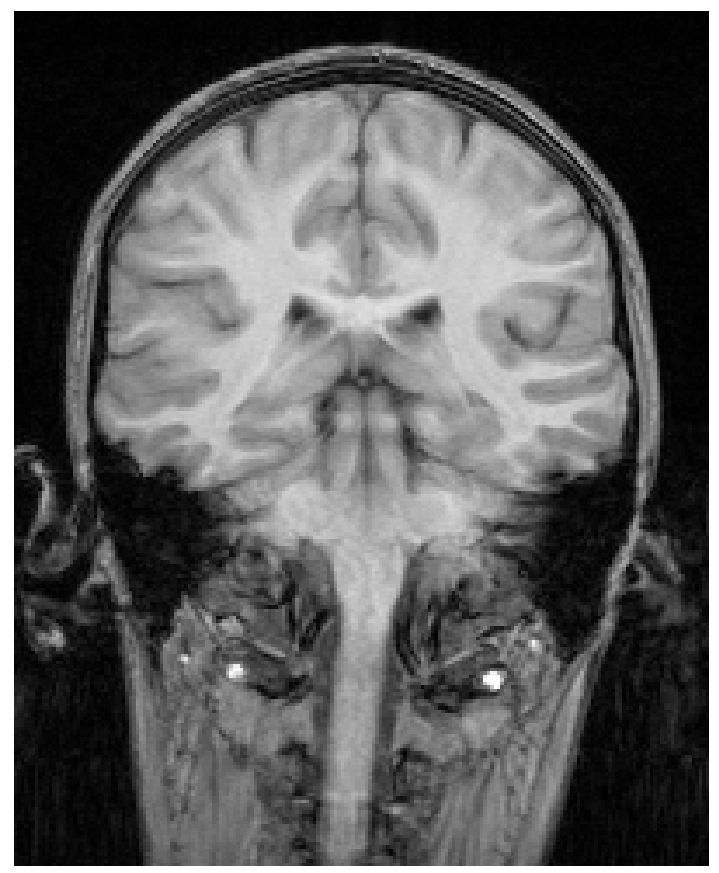

Fig. 3. Magnetic resonance image of human head (coronal section)

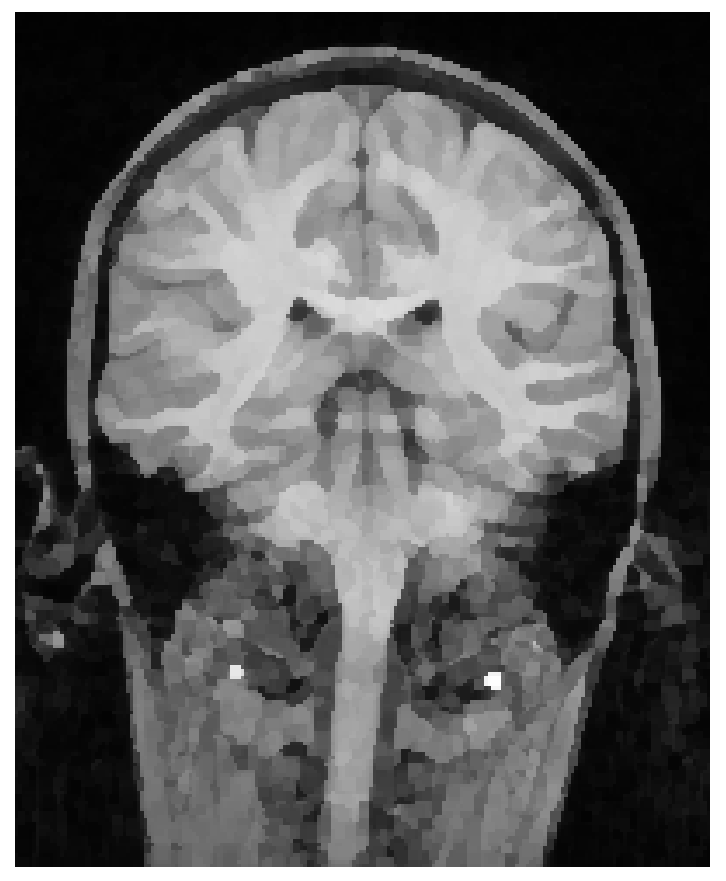

Fig. 5. Image filtered by the MLV filter ( $3 \times 3$ structuring element)

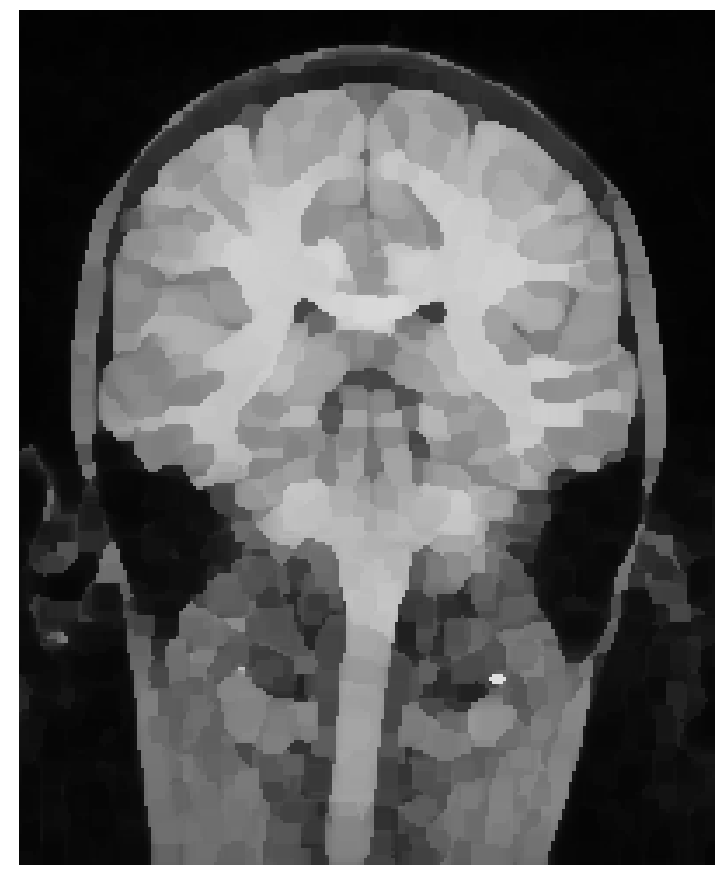

Fig. 4. Image filtered by the MLV filter $(5 \times 5$ structuring element $)$

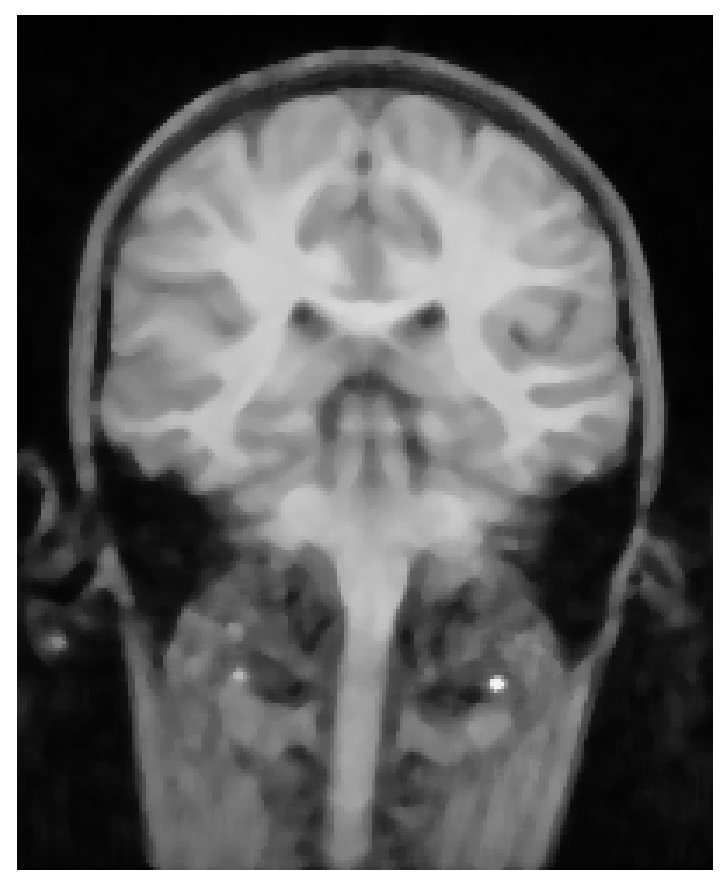

Fig. 6. Image filtered by a $5 \times 5$ square median filter 